\title{
When vestibular rehabilitation can assist: findings with use of data mining
}

\author{
Dimitrios Gatsios, Kostas M. Tsiouris, \\ Dimitrios I. Fotiadis \\ Unit of Medical Technology and \\ Intelligent Information Systems \\ University of Ioannina \\ Ioannina, Greece \\ fotiadis@cc.uoi.gr
}

\author{
Christoph Maurer \\ Department of Neurology \\ University of Freiburg \\ Freiburg, Germany \\ christoph.maurer@uniklinik- \\ freiburg.de
}

\author{
Dimitrios Kikidis, Athanasios Bibas, \\ Christos Nikitas \\ First Department of Otolaryngology- \\ Head and Neck Surgery, 'Hippokrateio' \\ General Hospital \\ National Kapodistrian University of \\ Athens \\ Athens, Greece \\ dimitriskikidis@yahoo.com
}

Floris L. Wuyts, Laura Celis

Lab for Equilibrium Investigations and Aerospace

University of Antwerp

Antwerp, Belgium

floris.wuyts@uantwerpen.be
Doris Eva Bamiou, Linda Luxon UCL Ear Institute \& University College London Hospitals Biomedical Research Centre Hearing \& Deafness

London, United Kingdom

d.bamiou@ucl.ac.uk

Leen Maes

Department of Rehabilitation Sciences

Ghent University

Ghent, Belgium

leenk.maes@ugent.be

Themis Exarchos

Department of Informatics

Ionian University

Corfu, Greece

exarchos@ionio.gr

\begin{abstract}
Falls, caused by dizziness and balance deficits, and their consequences in terms of the resultant functional limitations and of the associated costs for the involved actors as well as for the healthcare system are a serious problem in modern societies. Vestibular rehabilitation therapy (VRT) is proposed as a personalized intervention both for ameliorating symptoms and for improving balance. The outcomes of the therapy are not the same in all patients and the reasons are explored in this study with data mining methods applied in two retrospective datasets. According to our findings, patients with unstable lesions, concurrent psychological problems and with headaches are poorer candidates for VRT. Patients with stable vestibular deficits, of recent onset and relatively intact psychological, acoustic, visual and proprioceptive systems are better candidates for VRT.
\end{abstract}

\section{Keywords—vestibular rehabilitation, data mining}

\section{INTRODUCTION}

More than one third of the population will experience dizziness at least once in their lifetime. Some individuals develop permanent balance deficits. For elderly individuals (over 65 years) dizziness is a very common reason for visiting a physician and a significant risk factor for falls. Falls have been estimated to be the leading cause of serious injury and death in older citizens. Early, personalized treatment can prevent falls.

Vestibular rehabilitation therapy (VRT) [1] for persons suffering from dizziness and balance impairments has been shown to be effective [2]. VRT is designed to boost central vestibular compensation [3], decreases duration and intensity of symptoms such as dizziness and postural instability [4], reduces visual dependency (initially caused by deregulation after vestibular failure) [5] and lessens the number of falls and fear of falling [6, 7], as well as dizziness related distress, while increasing functional ability and Quality of Life (QoL) in individuals with vestibular disorders through the re-weighting of sensory inputs. The most beneficial way of maximizing improvements is via a customized and supervised rehabilitation protocol rather than a generalized exercise regime $[8,9]$.

Vestibular rehabilitation is a safe, effective management for unilateral peripheral vestibular dysfunction and is currently considered the best available option to resolve symptoms and improve functioning in the medium term [4]. Recently, clinical practice guidelines for peripheral vestibular hypofunction and benign paroxysmal positional vertigo provide the option that clinicians may offer self-administered vestibular rehabilitation $[10,11]$.

Still, VRT does not always provide full relief of symptoms and the outcomes differ from patient to patient even when they have the same diagnosis and similar symptoms. In this study, we analyze two retrospective datasets with data mining techniques to further explore the factors that may affect recovery and lead to poor outcomes in order to provide additional evidence to the clinicians and support them in deciding which patients should undergo vestibular rehabilitation and indicate the likely prognosis when VRT is prescribed.

Most of the previous work has been aimed at the application of data mining methods for the classification of patients in diagnostic groups [12-15]. This study aims to apply data mining techniques to explore factors (age, symptom severity, diagnosis etc.) that might affect the outcome of the VRT intervention negatively, i.e. focuses on prognosis.

\section{METHODS}

\section{A. EMBalance Dataset}

This retrospective dataset, that was built for the development of the EMBalance Decision Support System (DSS) [16], includes data from 1010 patients diagnosed with peripheral and central vestibular disorders (unilateral peripheral dysfunction/failure, psychological disorders, bilateral vestibular failure/dysfunction, cerebellar/pontine lesion, acoustic neuroma, chronic subjective dizziness, persistent postural-perceptual dizziness (PPPD), vestibular neuritis, Menière's disease, migrainous vertigo, vestibular 
paroxysmia, typical and atypical posterior benign paroxysmal positional vertigo (BPPV), that were collected from the National Hospital for Neurology and Neurosurgery, Queen Square, UK, the 1st Department of Otolaryngology, National Kapodistrian University of Athens (NKUA), Greece, the University of Antwerp, Belgium and the University Clinic of Freiburg, Germany. These data contained more than 160 features (variables), including patients' demographics, medical and disease related history, clinical and laboratory examination results (including auditory tests), treatment (besides medication) and medication (see Table I). Moreover, detailed information on symptoms related to the condition, symptoms duration, symptom free intervals, association between symptoms and relevant triggers were included.

\section{B. NKUA Dataset}

This retrospective dataset includes data from 200 patients diagnosed with peripheral and central vestibular disorders that were collected from NKUA, Greece. These data contained: Patient age/gender, duration of symptoms and diagnosis at baseline (unilateral peripheral dysfunction/failure, psychological disorders, bilateral vestibular failure/ dysfunction, cerebellar/pontine lesions, acoustic neuroma, chronic subjective dizziness, persistent postural-perceptual dizziness (PPPD), vestibular neuritis, Menière's disease, vestibular migraine, vestibular paroxysmia, typical and atypical posterior benign paroxysmal positional vertigo (BPPV), and measurements of the most commonly parameters used in the literature outcome measures, the Dizziness Handicap Inventory (DHI) [17] and the Functional Gait Assessment (FGA) [18] scores at baseline and at follow up.

\section{Evaluation of vestibular rehabilitation outcomes}

In both the EMBalance and the NKUA datasets the case explored was whether the symptoms were improved or not with vestibular rehabilitation being part of the treatment. In the EMBalance dataset the evaluation of the vestibular rehabilitation outcomes was performed within two consecutive visits, comparing patient's evaluation outcomes between the first visit and the follow-up visit to assess the response to treatment (i.e. patient's condition improved or not improved). In the NKUA dataset the difference in pre- and post-rehabilitation scores of 18 points for DHI [17] was used in order to define improvement.

\section{Data pre-processing}

The EMBalance dataset has great heterogeneity in the recorded data, since the aim during development was not focused on the evaluation of vestibular rehabilitation outcomes as was the case in the NKUA dataset. Thus, some refinement of data from the EMBalance dataset was undertaken to enable feature selection and classification for this study. Initially, patients who had not performed vestibular rehabilitation during their treatment were removed, as were patients who had performed vestibular rehabilitation but the outcome of their treatment was not evaluated or reported to ensure that there were no missing values in the class attribute. The data of the remaining patients were then reviewed to remove patients and features with many missing values or features that had a single, unique value across all cases, as they had no class discrimination potential. Finally, manual inspection of the remaining features was also performed to remove those which were irrelevant to the classification task (e.g. patient IDs, evaluation centers, dates, etc.).

Table I: Extracted features for the evaluation of vestibular rehabilitation outcomes.

\begin{tabular}{|l|c|}
\hline \multicolumn{2}{|c|}{ EMBalance dataset features } \\
\hline \multicolumn{1}{|c|}{ Broad category } & Number of features \\
\hline Patient demographics & 2 \\
\hline Clinical examination & 2 \\
\hline Vestibular tests & 14 \\
\hline Auditory tests & 3 \\
\hline Ear intervention & 5 \\
\hline Disabilities & 24 \\
\hline Symptoms & 7 \\
\hline Autonomic symptoms & 14 \\
\hline Vertigo triggering symptoms & 27 \\
\hline Medication & 11 \\
\hline Other treatment & 22 \\
\hline Disease diagnosis & \\
\hline & \\
\hline $\begin{array}{l}\text { Age, Gender, Duration of symptoms, Diagnosis, DHI score (baseline), } \\
\text { FGA score (baseline) }\end{array}$ \\
\hline
\end{tabular}

Applying the above methodology, resulted in a version of the EMBalance dataset that included 247 patients with vestibular rehabilitation as a module of their treatment plan, with 147 patients characterized as "Improved" and 100 patients as "Not improved". The number of features was reduced to 146 . The NKUA dataset did not require any preprocessing and the evaluation dataset included all 200 patients, with 136 cases characterized as "Improved" and 64 as "Not improved", respectively, with six features being available. The extracted features for both datasets are reported in more detail in Table I.

\section{E. Feature selection and classification}

An extensive search was performed in the available feature space for each dataset (Table I) to isolate the most informative features suggestive of a poor response to vestibular rehabilitation, using the best-first search algorithm. The best-first is an iterative algorithm starting from single feature nodes and adding an extra feature at a time, in the search for an optimal subset of features that maximizes classification accuracy. Nodes continue to expand with more features added at each step until there is no increase in classification performance using the training instances. Then the subset of features that offered the highest accuracy using the minimum amount of features is selected for validation with the testing dataset. For a more robust evaluation, 10-fold cross-validation is performed by splitting the available instances into ten groups, using one for testing and the remaining for training interchangeably.

For the actual classification process the Repeated Incremental Pruning to Produce Error Reduction, RIPPER algorithm is used. RIPPER is a rule learning-based classifier which provides classification rules that can be easily interpreted in human language ("If (condition1) and/or (condition2) then class $=\ldots$.."). The advantage of 
obtaining rules that are easy to interpret is that the reasoning behind the classification can be assessed by nonmachine learning experts, such as the clinical users targeted in this study, adding more value to the extracted feedback. Finally, in cases where there is significant difference between the number of instances of the two classes, cost-sensitive classification is used to negate the effects of class imbalance in classification performance. The classifier is developed using the Weka API, an open source collection of machine learning algorithms for data mining, issued under the GNU General Public License.

\section{RESULTS}

In order to consider any given classification results as significant and informative, a sensitivity and specificity $>=$ 0.7 was required in both cases. The extracted rules are provided in terms of classification significance using the convergence to false positive ratio: e.g. (100.0/11.0) means that the rule applied to 100 patients with 11 false positives and, thus, 89 true positives. Classification rules with low impact were omitted to keep the size of the presented results within reason. Below we present the most significant classification results for each dataset.

\section{A. EMBalance analysis results}

The factors that seemed to be associated with deterioration of symptoms despite ongoing or completed VRT and their significance are depicted in Table II. We notice that diagnosis of psychological disorders, of Migraine Vertigo (MV) and of Unilateral Peripheral Vestibular Dysfunction (UPVD) is suggestive of suboptimal results from VRT. Moreover, symptoms such as headaches and hearing loss can also be associated with poor VRT results, while symptoms very common in bilateral vestibulopathy (BVP) such as having difficulty walking in darkness or on uneven surfaces and oscillopsia can also be associated with the effectiveness of VRT.

Table II: EMBalance analysis results.

\begin{tabular}{|l|c|c|}
\hline \multicolumn{3}{|c|}{ EMBalance Classification Rules } \\
\hline \multicolumn{1}{|c|}{ Fases } & $\begin{array}{c}\text { False } \\
\text { Positives }\end{array}$ \\
\hline Diagnosis of Psychological Disorders & 19.8 & 0.5 \\
\hline $\begin{array}{l}\text { Diagnosis of Migrainous vertigo (Vestibular } \\
\text { Migraine) }\end{array}$ & 10.3 & 1.7 \\
\hline $\begin{array}{l}\text { Diagnosis of Unilateral Peripheral Vestibular } \\
\text { Dysfunction/Failure }\end{array}$ & 69.5 & 22.7 \\
\hline Having headache as symptom & 91.1 & 29.5 \\
\hline Having hearing loss in both ears & 20.7 & 7.1 \\
\hline Having difficulty walking in darkness & 20.7 & 7.1 \\
\hline Having difficulty walking on uneven surfaces & 22.3 & 8.7 \\
\hline Having oscillopsia & 4.0 & 0.0 \\
\hline
\end{tabular}

\section{B. NKUA analysis results}

The factors that seemed to be associated with deterioration of symptoms despite VRT in the NKUA dataset are the early onset of symptoms and the limited postural stability during walking, as depicted in Table III.

Table III: NKUA analysis results.

\begin{tabular}{|l|l|c|}
\hline \multicolumn{3}{|c|}{ NKUA Classification Rules } \\
\hline Factor & Cases & $\begin{array}{c}\text { False } \\
\text { Positives }\end{array}$ \\
\hline
\end{tabular}

\begin{tabular}{|c|c|c|}
\hline \multicolumn{3}{|c|}{ NKUA Classification Rules } \\
\hline \multicolumn{1}{|c|}{ Factor } & Cases & $\begin{array}{c}\text { False } \\
\text { Positives }\end{array}$ \\
\hline $\begin{array}{l}\text { Duration of symptoms }>=24 \text { months AND } \\
\text { FGA at baseline }<=23\end{array}$ & 14.0 & 0.0 \\
\hline
\end{tabular}

\section{DISCUSSION}

Firstly, it should be noted that the associations presented in Tables II and III do not necessarily imply causation and the discussion of findings is not straightforward in such retrospective datasets, as there is a number of potential confounders. From the datasets themselves we note that not all patients undergoing vestibular rehabilitation as a module of their management plan improve. In fact, in the NKUA dataset $68 \%$ of the patients (136 of 200) improved at discharge.

The clinical practice guidelines [10] are consistent about the factors that negatively affect the outcomes of vestibular rehabilitation, including longer duration of symptoms (Table III), patient comorbidities such as migraine and psychological factors such as anxiety (Table II). Comorbid psychologic disorders (neuroticism, introversion, catastrophizing) can have a negative effect in rehabilitation and are associated with worse outcomes [19]. Furthermore, research supports the use of psychological input to complement vestibular rehabilitation in an effort to achieve better outcomes for patients with anxiety [20].

The findings suggest that diagnosis of vestibular migraine is indicative of overall poor outcomes from vestibular rehabilitation. In our study this may be attributed to the fact that we do not compare outcomes at discharge but at varying timepoints for different patients. Still, these findings are consistent with the availability of weak data (no current randomized trials on efficacy) supporting the effectiveness of VRT in the management of vestibular migraine [21], [22] and thus more evidence is required to establish an effect. Vestibular migraine patients are likely to require longer follow up and longer time to improve. Subcategorizing migraineurs based on number of episodes per month, duration and intensity of symptoms and levels of anxiety, as well as investigating optimal dosage of visual exposure could be the future clinical questions for the improvement of the effectiveness.

The finding that the diagnosis of unilateral peripheral vestibular dysfunction/failure may be suggestive of poor outcomes from VRT is in contrast to recent reviews which report moderate to strong evidence that vestibular rehabilitation is a safe, effective management for UPVD [23]. Sensory and/or visual dependency that may result from dysfunction/failure could be considered as a strong factor for poor improvement. This finding is interesting and will be studied more in future studies from the group.

Moreover, vestibular rehabilitation has been found to improve headache in patients with vestibular migraine and in patients with dizziness and tension-type headache [24]. Even, in this study the improvement in dizziness was more significant in patients with VM than those with comorbid tension-type headache which is consistent with our findings since headache in our dataset also included comorbid tension-type. Furthermore, the symptoms difficulty of walking in darkness, difficulty walking on uneven surfaces and oscillopsia are the major symptoms of BVP [25]. VRT is indeed useful for treating chronic BVP, 
but the reasons why a limited number of patients show improvement are to be defined [26].

Overall, poor results from VRT may be attributed to multiple biological, psychological and social factors which are affecting the medical profile of patients and therefore their behavior towards symptoms. From a clinicians' perspective, poor outcome could be due to the lack of optimal exercise dosage, low levels and form of supervision, lack of motivation and inappropriate forms and level of rewards. Novel technological achievements towards real time monitoring and mixed reality environments are promising for enhanced adherence and compliance.

\section{CONCLUSIONS}

We have shown that data mining can be useful for analyzing retrospective datasets and confirm findings for VRT that agree with recent literature and more focused clinical studies exploring specific aspects of VRT.VRT is traditionally a good choice for patients with unchanging symptomatic vestibular deficits that have not resolved with spontaneous vestibular compensation. In fact, patients with unstable lesions, i.e. those with fluctuating symptoms of imbalance, those with mental comorbidities (especially anxiety) and those having headaches as a comorbid symptom are not good candidates for VRT. Patients considered for VRT should have stable central or peripheral vestibular deficits, of recent onset and as relatively intact psychological, acoustic, visual and proprioceptive systems.

\section{ACKNOWLEDGMENT}

This work has received funding from the European Union's Horizon 2020 research and innovation programme under grant agreement No 769574.

\section{REFERENCES}

[1] S. L. Whitney, A. A. Alghwiri, and A. Alghadir, "An overview of vestibular rehabilitation," Handb Clin Neurol, vol. 137, pp. 187-205, 2016.

[2] P. M. Dunlap, J. M. Holmberg, and S. L. Whitney, "Vestibular rehabilitation: advances in peripheral and central vestibular disorders," Curr Opin Neurol, vol. 32, no. 1, pp. 137-144, Feb 2019

[3] A. M. Bronstein, T. Lempert, and B. M. Seemungal, "Chronic dizziness: a practical approach," Pract Neurol, vol. 10, no. 3, pp. 129-39, Jun 2010.

[4] M. N. McDonnell and S. L. Hillier, "Vestibular rehabilitation for unilateral peripheral vestibular dysfunction," Cochrane Database Syst Rev, vol. 1, p. CD005397, Jan 132015.

[5] M. Pavlou, "The use of optokinetic stimulation in vestibular rehabilitation," J Neurol Phys Ther, vol. 34, no. 2, pp. 105-10, Jun 2010.

[6] S. J. Herdman, M. C. Schubert, and R. J. Tusa, "Strategies for balance rehabilitation: fall risk and treatment," Ann NY Acad Sci, vol. 942, pp. 394-412, Oct 2001.

[7] J. D. Macias, S. Massingale, and R. D. Gerkin, "Efficacy of vestibular rehabilitation therapy in reducing falls," Otolaryngol Head Neck Surg, vol. 133, no. 3, pp. 323-5, Sep 2005.

[8] T. Szturm, D. J. Ireland, and M. Lessing-Turner, "Comparison of different exercise programs in the rehabilitation of patients with chronic peripheral vestibular dysfunction," J Vestib Res, vol. 4, no. 6, pp. 461-79, NovDec 1994.

[9] M. B. Badke, T. A. Shea, J. A. Miedaner, and C. R. Grove, "Outcomes after rehabilitation for adults with balance dysfunction," Arch Phys Med Rehabil, vol. 85, no. 2, pp. 227-33, Feb 2004.

[10] C. D. Hall et al., "Vestibular Rehabilitation for Peripheral Vestibular Hypofunction: An Evidence-Based Clinical Practice Guideline: FROM THE AMERICAN PHYSICAL THERAPY ASSOCIATION NEUROLOGY SECTION," J Neurol Phys Ther, vol. 40, no. 2, pp. 124-55, Apr 2016.

[11] N. Bhattacharyya et al., "Clinical Practice Guideline: Benign Paroxysmal Positional Vertigo (Update)," Otolaryngol Head Neck Surg, vol. 156, no. 3_suppl, pp. S1-S47, Mar 2017.

[12] N. Chawla and J. S. Olshaker, "Diagnosis and management of dizziness and vertigo," Med Clin North Am, vol. 90, no. 2, pp. 291-304, Mar 2006.

[13] M. Siermala, M. Juhola, and E. Kentala, "Neural network classification of otoneurological data and its visualization," Comput Biol Med, vol. 38, no. 8, pp. 858-66, Aug 2008.

[14] K. Miettinen and M. Juhola, "Classification of otoneurological cases according to Bayesian probabilistic models," J Med Syst, vol. 34, no. 2, pp. 119-30, Apr 2010.

[15] T. P. Exarchos et al., "Mining balance disorders' data for the development of diagnostic decision support systems," Comput Biol Med, vol. 77, pp. 240-8, Oct 12016.

[16] L. Rammazzo et al., "EMBalance - validation of a decision support system in the early diagnostic evaluation and management plan formulation of balance disorders in primary care: study protocol of a feasibility randomised controlled trial," Trials, vol. 17, no. 1, p. 435, Sep 52016.

[17] G. P. Jacobson and C. W. Newman, "The development of the Dizziness Handicap Inventory," Arch Otolaryngol Head Neck Surg, vol. 116, no. 4, pp. 424-7, Apr 1990.

[18] D. M. Wrisley, G. F. Marchetti, D. K. Kuharsky, and S. L. Whitney, "Reliability, internal consistency, and validity of data obtained with the functional gait assessment," Phys Ther, vol. 84, no. 10, pp. 906-18, Oct 2004.

[19] F. Goto, K. Nomura, F. Taka, M. Arai, and N. Sugaya, "Analysis of Factors Affecting the Outcomes of Inhospitalized Vestibular Rehabilitation in Patients With Intractable Dizziness," Otol Neurotol, vol. 38, no. 3, pp. 368-372, Mar 2017.

[20] M. Dieterich and J. P. Staab, "Functional dizziness: from phobic postural vertigo and chronic subjective dizziness to persistent postural-perceptual dizziness," Curr Opin Neurol, vol. 30, no. 1, pp. 107-113, Feb 2017.

[21] C. G. Lauritsen and M. J. Marmura, "Current Treatment Options: Vestibular Migraine," Curr Treat Options Neurol, vol. 19, no. 11, p. 38, Sep 302017.

[22] A. H. Alghadir and S. Anwer, "Effects of Vestibular Rehabilitation in the Management of a Vestibular Migraine: A Review," Front Neurol, vol. 9, p. 440, 2018.

[23] S. Hillier and M. McDonnell, "Is vestibular rehabilitation effective in improving dizziness and function after unilateral peripheral vestibular hypofunction? An abridged version of a Cochrane Review," Eur J Phys Rehabil Med, vol. 52, no. 4, pp. 541-56, Aug 2016.

[24] N. Sugaya, M. Arai, and F. Goto, "Is the Headache in Patients with Vestibular Migraine Attenuated by Vestibular Rehabilitation?," Front Neurol, vol. 8, p. 124, 2017.

[25] F. Porciuncula, C. C. Johnson, and L. B. Glickman, "The effect of vestibular rehabilitation on adults with bilateral vestibular hypofunction: a systematic review," J Vestib Res, vol. 22, no. 5-6, pp. 283-98, 2012.

[26] N. Lehnen et al., "Head-Movement-Emphasized Rehabilitation in Bilateral Vestibulopathy," Front Neurol, vol. 9, p. 562, 2018. 